\title{
Unsteady Interaction Between a Transonic Turbine Stage and Downstream Components
}

\author{
Roger L. Davis \\ University of California-Davis, Davis, California, USA
}

Jixian Yao

General Electric, Corporate R\&D Center, Niskayuna, New York, USA

John P. Clark

Turbine Research Facility, Air Force Research Lab, Dayton, Ohio, USA

Gary Stetson

Pratt \& Whitney, E. Hartford, Connecticut, USA

Juan J. Alonso and Antony Jameson

Stanford University, Stanford, California, USA

Charles W. Haldeman and Michael G. Dunn

Gas Turbine Laboratory, Ohio State University, Columbus, Ohio, USA

Results from a numerical simulation of the unsteady flow through one quarter of the circumference of a transonic high-pressure turbine stage, transition duct, and lowpressure turbine first vane are presented and compared with experimental data. Analysis of the unsteady pressure field resulting from the simulation shows the effects of not only the rotor/stator interaction of the high-pressure turbine stage but also new details of the interaction between the blade and the downstream transition duct and low-pressure turbine vane. Blade trailing edge shocks propagate downstream, strike, and reflect off of the transition duct hub and/or downstream vane leading to high unsteady pressure on these downstream components. The reflection of these shocks from

Received 14 January 2003; accepted 4 April 2004.

The authors would like to thank the U.S. Department of Energy (DoE) for its generous support under the ASCI program. The authors would especially like to recognize the support of the staff at the Lawrence Livermore National Laboratory. We would also like to thank the managers at United Technologies Research Center and Pratt \& Whitney for their support.

Address correspondence to Roger L. Davis, Department of Mechanical and Aeronautical Engineering, University of California, Davis, One Shields Avenue, 2104 Bainer Hall, Davis, CA 95616, USA. E-mail: davisr1@ucdavis.edu the downstream components back into the blade itself has also been found to increase the level of unsteady pressure fluctuations on the uncovered portion of the blade suction surface. In addition, the blade tip vortex has been found to have a moderately strong interaction with the downstream vane even with the considerable axial spacing between the two blade-rows. Fourier decomposition of the unsteady surface pressure of the blade and downstream low-pressure turbine vane shows the magnitude of the various frequencies contributing to the unsteady loads. Detailed comparisons between the computed unsteady surface pressure spectrum and the experimental data are shown along with a discussion of the various interaction mechanisms between the blade, transition duct, and downstream vane. These comparisons show overall good agreement between the simulation and experimental data and identify areas where further improvements in modeling are needed.

Keywords Turbomachinery, Aeroelasticity, Forced response, High-
cycle fatigue, Unsteady flow

\section{INTRODUCTION}

Significant experimental and numerical research has been performed over the last several years on highlighting and understanding the unsteady aerodynamics of multi-stage 
turbomachinery (e.g., Reinmoller et al., 2001; Arnone et al., 2001; Gombert and Hohn, 2001; Hohn, 2001; Dorney et al., 2001; Clark et al., 2000; Busby et al., 1998; Rao et al., 1994; Chen et al., 1994; Sharma et al., 1992; Takahashi and Ni, 1991; Giles, 1990; Jorgenson and Chima, 1990; Rai, 1987; Dring et al., 1992). Much of this work has focused on quantifying the unsteady flow field resulting from the interaction between rotors and stators in terms of aerodynamic performance and airfoil surface unsteady pressure. Losses in aerodynamic performance due to unsteady flows can be significant and the amplitude and frequency of the unsteady airfoil surface pressure can result in excessive stress levels that result in premature fatigue. The drive to remove weight and cost from the engine has pushed designs toward single-stage, transonic high-pressure turbines. The rotor/stator interaction of transonic turbine stages has been of particular interest recently because of the additional timeaverage losses and unsteady interactions caused by the trailing edge shock systems that exist at supersonic exit conditions (e.g., Clark et al., 2000; Busby et al., 1998). Another factor recently brought to light in transonic high-pressure turbine stages is the interaction of the trailing edge shock system with transition ducts and the vanes of the downstream low pressure turbine. These downstream components can produce significant reflections of the high-pressure turbine shocks back upstream into the high-pressure turbine itself causing large unsteady pressure amplitudes and possible stress problems. Also, recent numerical investigations (Clark et al., 2000; Yao et al., 2002) have also shown the importance of modeling actual blade counts in unsteady-flow turbine multi-stage simulations for accurate prediction of both entropy migration and the unsteady frequency spectrum resulting from rotor/stator interaction.

In an effort to drive toward large-scale, multi-component, multi-physics simulation capability, the Department of Energy (DoE) launched the Accelerated Strategic Computing Initiative (ASCI) Program. The goal of this program is to promote the application and development of massively parallel computer systems for the solution of large-scale engineering and science problems. The current investigation, which is a part of the DoE ASCI program, is focused on developing and demonstrating large-scale simulation capability for flows in gas-turbine engines (see Reynolds et al., 2001). Specifically, the current effort has been focused on demonstrating the capability to compute unsteady flows in multi-stages of a turbine and illustrating the flow physics associated with high-/low-pressure turbine interaction.

This article presents the results from a numerical simulation of unsteady flow through a single-stage, high-pressure turbine, transition duct, and downstream low-pressure turbine vane. These results are compared with experimental time-averaged and unsteady surface pressure data. In order to obtain accurate predictions of the loss migration and unsteady surface pressure, one quarter of the circumference of the machine is simulated. Details of the shock/blade/transition duct interaction are discussed along with an analysis of the unsteady pressure spectrum. The predicted time-average and unsteady surface pressure distributions are compared with experimental data. These results highlight the flow physics and shock reflections from the downstream transition duct and low-pressure turbine first vane that lead to the unsteady surface pressure on the high-pressure turbine blade. Finally, the computed time-average relative total pressure loss for each blade-row is compared with that from a steady, multi-stage analysis to quantify the unsteady effects on aerodynamic performance.

\section{CONFIGURATION}

Figure 1 shows the high-pressure turbine stage, transition duct, and low-pressure turbine first vane that was tested experimentally at the Ohio State University Gas Turbine Laboratory (Dunn et al., 1989; Clark et al., 2000) and used in the present numerical investigation. This geometry is representative of a modern high-pressure turbine design consisting of 36 and 56 high-pressure turbine vanes and blades, respectively, and 36 lowpressure turbine first vanes. The simulation used $1 / 4$ of these counts in order to reduce the simulation size but yet maintain the fundamental periodicity of the configuration. This led to a 9-vane1, 14-blade, 9-vane2 model for the simulation. This model not only allowed for geometrical similitude between simulation and experiment but also allowed comparisons to be made between the predictions performed here with those by Clark et al. (2000).

The initial investigation by Clark et al. (2000) showed the effect of blade-count and airfoil scaling on the unsteady pressure field for this configuration. Their study showed that if airfoil scaling was used on the blade (holding midspan axial gap) to reduce the simulation to a 2-vane1, 3-blade, 2-vane2 model

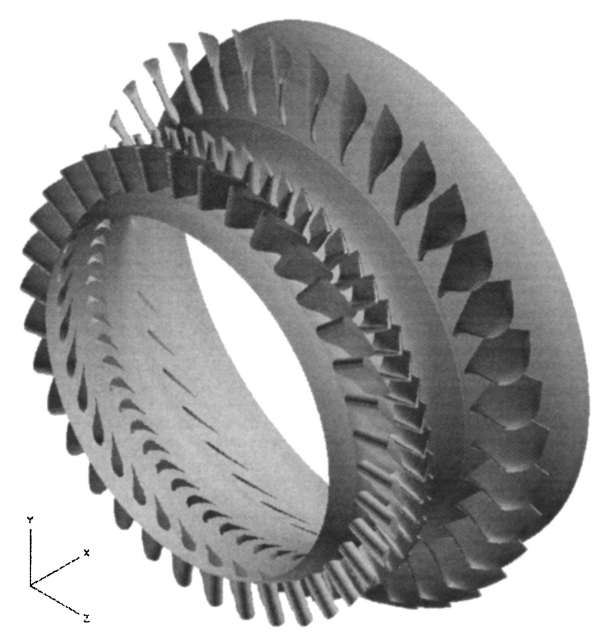

$$
\text { Configuration }
$$

FIGURE 1

High-pressure turbine stage, transition duct, 1st vane low-pressure turbine configuration. 
(1/18th of the wheel), errors in the unsteady surface pressure amplitudes could arise. It was pointed out that these errors could lead to under- or over-prediction of resonant stresses on excited blades. Similar adverse effects of airfoil scaling were found in the investigation of Yao et al. (2002) for a subsonic $11 / 2$ stage turbine. In addition to showing the importance of modeling the actual blade-count, the simulation results of Clark et al. (2000) showed some lack in agreement between their 9-vane1, 14-blade, 9-vane2 (1/4th of the wheel) predicted time-average and unsteady pressures and the experimental data near the hub and the tip of the blade. Unfortunately, no comparisons between their predictions and the experimental data were shown for the downstream second vane. The current investigation was performed as a validation of the TFLO (Yao et al., 2001) procedure, an independent verification of the results of Clark et al. (2000), as well as a grid-refinement study to see if grid-density was a major factor in prediction accuracy. As described below, the computational grid density used in the current investigation was approximately three times greater than that used by Clark et al. (2000). Finally, the focus of the current investigation has been on the interaction between the blade, transition duct, and downstream low-pressure turbine vane, which has not been previously described.

The flow conditions used for this investigation correspond to a design point corrected flow parameter of $7.69 \times 10^{-4}(\mathrm{~kg}$ $\mathrm{K}^{1 / 2} /(\mathrm{Pa}$ s). A uniform total pressure, total temperature, and axial velocity (zero swirl) profile were held as the inlet boundary condition in the simulation. The exit static pressure profile measured in the rig was held as the exit boundary condition. The inlet and exit boundary conditions were set at the design-intent conditions established from a through-flow analysis. The inlet total to exit static pressure ratio was 5.19 for the simulation (design intent) and 5.17 for the experiment. The corrected speed parameter $\left(\mathrm{rpm} / \mathrm{K}^{1 / 2}\right)$ was 421 for the simulation and 419 for the experiment. No blade/hub leakage flows were included in the simulation. Further details of the flow conditions and rig geometry are provided by Clark et al. (2000).

Unsteady surface pressure measurements were taken at $10 \%$, $50 \%$, and $90 \%$ span (as measured from the hub) on all 3 bladerows of the rig. Details of the experimental measurement system and accuracy are reported in Clark et al. (2000) and Dunn et al. (1989).

Since a goal of the present study is to assess the predictive capability for magnitudes of unsteady forcing in a transonicturbine environment, every effort was made to ensure that the comparisons in the frequency domain between experimental and predicted results were consistent. For example, the numerical simulation was "sampled" over a time-interval for which the flow-field was periodic. This was the time over which a single blade traversed $90^{\circ}$, passing $1 / 4$ of the 36 vanes that composed the vane rings. This guaranteed that the passing frequencies of interest were evenly divisible by the spectral resolution of the sampled signal. The experimental data was in turn sampled for an interval corresponding to an integer number of rotor revolu- tions. Consequently, the passing frequencies in the experiment were also integer multiples of the experimental spectral resolution. In this way, picket fencing effects were avoided in both the predicted and experimental spectrum estimates. Also, although the experimental time-histories were nominally periodic on a once per revolution basis, random unsteadiness in the flow-field as well as electromagnetic pickup combined to ensure that the experimental signals were never truly periodic on any time interval. Therefore, the nominally periodic experimental signals were multiplied by a Hanning window-function to force periodicity in the data over the sample interval and thereby to avoid spectral leakage in the frequency-domain results (see Ifeachor and Jervis, 1996). However, the window function acted to broaden the peaks of interest in the spectrum estimates and reduce the amplitudes of the airfoil passing events by a factor of approximately 2.0. Therefore, the same window function was applied to the time histories predicted in the simulations even though they were truly periodic. In the results that follow, the authors concentrate on the Fourier-component magnitudes at the fundamental airfoil passing-frequency and its first harmonic. This is in keeping with the results of Clark et al. (2000) that showed that over $90 \%$ of the predicted signal power was contained in those frequencies for this turbine geometry under these flow-field conditions. The fundamental airfoil-passing frequencies and its first harmonic are both well below the experimental and computational Nyquist frequencies, and low-pass filters were employed in the experiment to avoid aliasing (Dunn et al., 1989).

\section{NUMERICAL SIMULATION}

The three-dimensional, multi-block, parallel flow solver, TFLO (Yao et al., 2001) has been developed under the DoE ASCI program in an effort to step up to large-scale parallel steadyand unsteady-flow multi-stage turbomachinery simulations. The unsteady Reynolds-averaged Navier-Stokes (RANS) equations are solved in the TFLO procedure using a cell-centered discretization on arbitrary multi-block meshes. The solution algorithm is based on an efficient implicit, dual time-step procedure (Jameson, 1991) in which an explicit Runge-Kutta integration scheme coupled with multi-grid, implicit residual smoothing, and local time-stepping convergence acceleration techniques are used in an inner iteration for each time-step. Wilcox's (1998) $k-\omega$ two-equation turbulence model is used to predict the turbulence viscosity in the field. The solver is parallelized using domain decomposition, a Single Program Multiple Data (SPMD) strategy, and the Message Passing Interface (MPI) standard.

The mesh sizes used in this calculation, in the axial $\times$ circumferential $\times$ radial directions, can be summarized as follows: 1 st vane: $209 \times 57 \times 73$, rotor: $217 \times 57 \times 73$ for the main passage, and $73 \times 17 \times 17$ for the tip gap; 2 nd vane: $281 \times 57 \times 73$. This resulted in approximately 31.3 million points. A total of 97,73 , and 97 points were used in the axial direction along the airfoil chord between the leading and trailing edges for the first vane, blade, and second vane, respectively. This grid density is 
approximately 3 times (63\% more streamwise, $16 \%$ more pitchwise, and $28 \%$ more spanwise) that used by Clark et al. (2000) and was chosen to determine the effect of grid resolution on the agreement between the unsteady surface pressure and the experimental data. Since a focus of the current investigation has been on the interaction between the blade and the downstream components, most of the additional grid density has been added in this region of the configuration.

The blade tip gap flow was solved as part of the overall simulation. The tip gap was uniform and equal to $1.15 \%$ of the blade span. Based upon numerical experience, the grid resolution of $73 \times 17 \times 17$ points has been found to adequately resolve the flow in this region. The computational grid is clustered near the blade tip and near the casing to resolve the high flow gradients and viscous flow in this region.

In order to accurately resolve the wave-forms corresponding to the stator-rotor-stator interaction, a total of 100 time steps were used to rotate one blade past one first vane passage. This resulted in the use of 1400 time steps for one global period corresponding to $1 / 4$ of the turbomachine circumference. For each time step, 30 multi-grid cycles were performed to ensure the convergence of the inner iteration. The use of 30 inner iterations for each time-step is not a general requirement but was chosen in the current investigation to ensure second-order temporal accuracy.

The 9-14-9 blade-count simulation was executed on 192 processors of the DoE Frost IBM SP3 at Lawrence Livermore Laboratory. The total memory required was approximately 16 gigabytes distributed over the 192 processors. Each time step (with 30 inner iterations) required approximately 16.5 minutes of clock time including the computational and restart file input/output time. The parallel computational efficiency of the TFLO code for up to 1024 processors is documented in Yao et al. (2001) demonstrating its linear scalibility. Two global cycles (or 18 vane passings by the blade or 28 blade passings by a vane) were executed to achieve a solution in which the airfoil unsteady surface pressures were time-periodic. Thus, the turn-around time for this simulation with the 192 IBM SP3 processors would be approximately one month.

\section{TIME-AVERAGED PRESSURE AND PRESSURE ENVELOPES}

The computed time-averaged and minimum/maximum pressure envelopes for the blade have been compared with experimental data reported by Clark et al. (2000). In addition, similar new comparisons are shown below for the downstream lowpressure turbine vane.

Figure 2 shows a comparison of the time-averaged surface pressures and the minimum/maximum pressure envelopes at $10 \%, 50 \%$, and $90 \%$ span postions from the unsteady simulation with the experimental data reported by Clark et al. (2000). This figure shows that the computed suction side pressure levels and envelopes, in general, compare very well with the experimen-

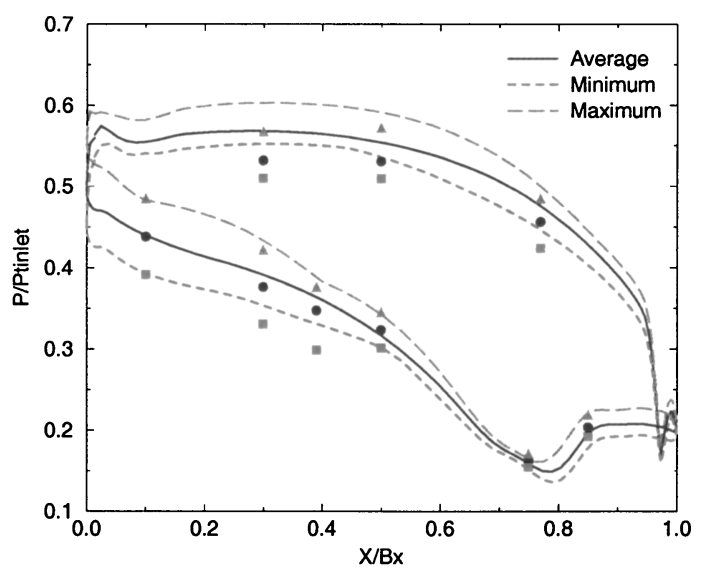

$10 \%$ Span

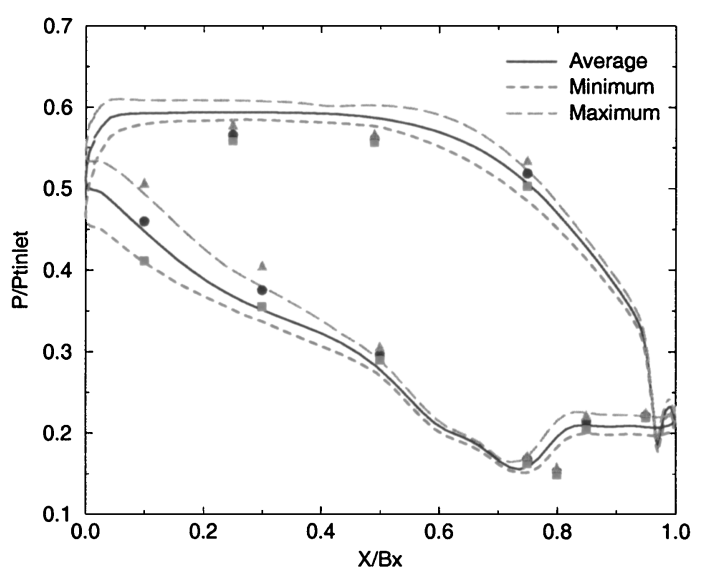

$50 \%$ Span

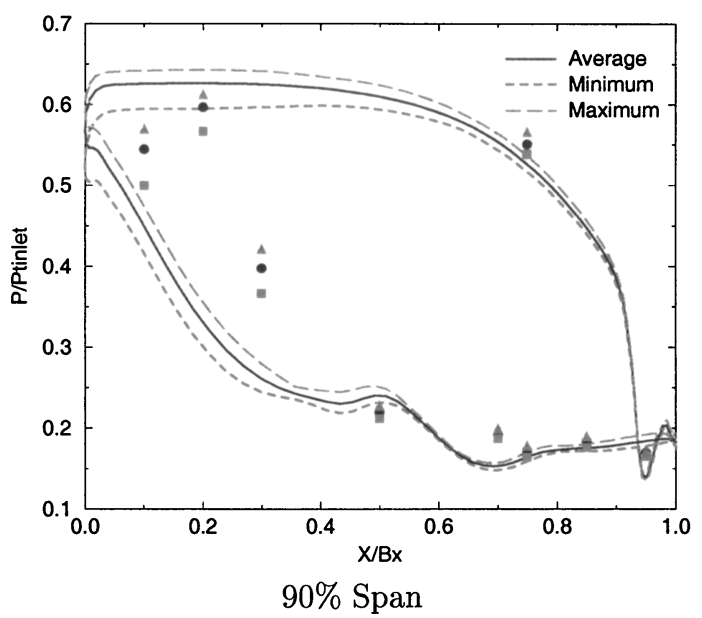

FIGURE 2

High-pressure turbine blade pressure distributions.

tal data at the $10 \%$ span and mid-span positions. The computed pressure levels are under-predicted on the suction side at the $90 \%$ spanwise position on the front half of the blade section, however. The computed pressure side envelopes are in good agreement with the experimental data but the computed pressure levels are 
high on the front half of the blade at all three spanwise positions. The predicted results shown by Clark et al. (2000) are very similar to those shown in Figure 2 leading to the possibility that either the blade shapes and/or the inlet profiles used in the simulations are slightly different than the experiment. As previously reported, the data at the $30 \%$ axial chord position on the suction side at the $90 \%$ spanwise position is suspect.

A similar comparison between the computed and the experimental time-averaged and minimum/maximum pressure envelopes for the low-pressure turbine vane is shown in Figure 3 (note that the pressure scale of this figure is different than that shown in Figure 2 to show more detail). The computed pressure levels and envelopes shown here are in better agreement with the experimental data than those previously predicted (but not shown) by Clark et al. (2001) indicating that either the increased computational grid density or different turbulence model has had a favorable effect. The absolute differences between the computed pressures and the experimental data for the low-pressure turbine vane are approximately the same as the differences observed for the blade (but appear larger due to the different pressure scales).

At $10 \%$ span, the pressure side pressure levels compare very well with the experimental data but the pressure envelope is predicted to be too large. This difference may be due to the lack of including blade leakage flows at the blade hub that exist in the experiment. The leakage flow, as a percentage of the mainstream flow in the experimental rig, leaving the mainstream and going into cavities just up- and downstream of the blade were estimated to be approximately $1 \%$ and $0.5 \%$, respectively. These leakage flows, although small in magnitude, could be responsible for reducing the boundary-layer thickness and flow unsteadiness downstream of the blade along the hub in the experiment. Also, as mentioned above, comparison of the present unsteady pressure results with those of Clark et al.'s (2000) for the low-pressure turbine vane show that agreement with the experimental data has definitely improved as a result of the increased computational grid density. However, since Wilcox's (1998) $k-\omega$ turbulence model was used in the present investigation compared to the Baldwin-Lomax (1978) algebraic model used by Clark et al. (2000), there is the possibility that the turbulence model could be responsible for some improvement.

On the suction side at the $10 \%$ span location, the pressure level and pressure envelope are overall in very good agreement with the experimental data. Only the pressure level at the $10 \%$ axial chord location is over-predicted. At mid-span, the computed pressure levels and envelope are in very good agreement with the experiment on both sides of the airfoil and at all axial locations. At $90 \%$ span, the pressure side pressure levels are in good agreement with the experiment but the envelope is predicted to be too great compared with the data. The pressure level of the experimental data at the $20 \%$ axial chord location seems obviously too low and is suspect. On the suction side at the $90 \%$ span location, the computed pressure levels are in good

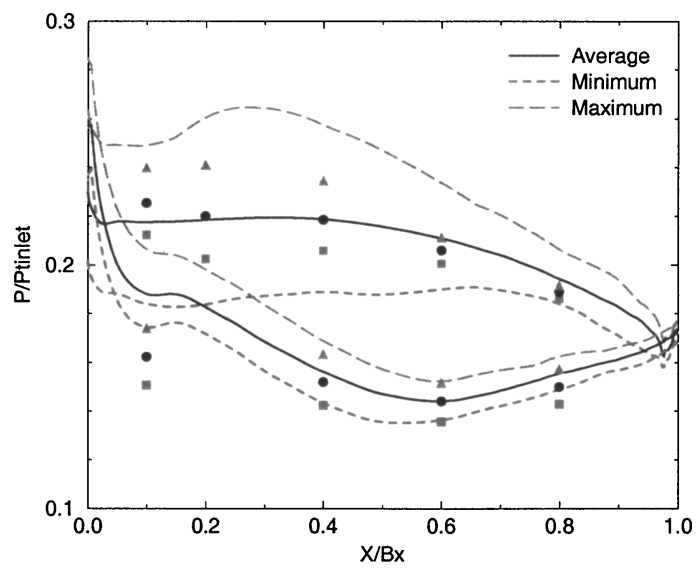

$10 \%$ Span
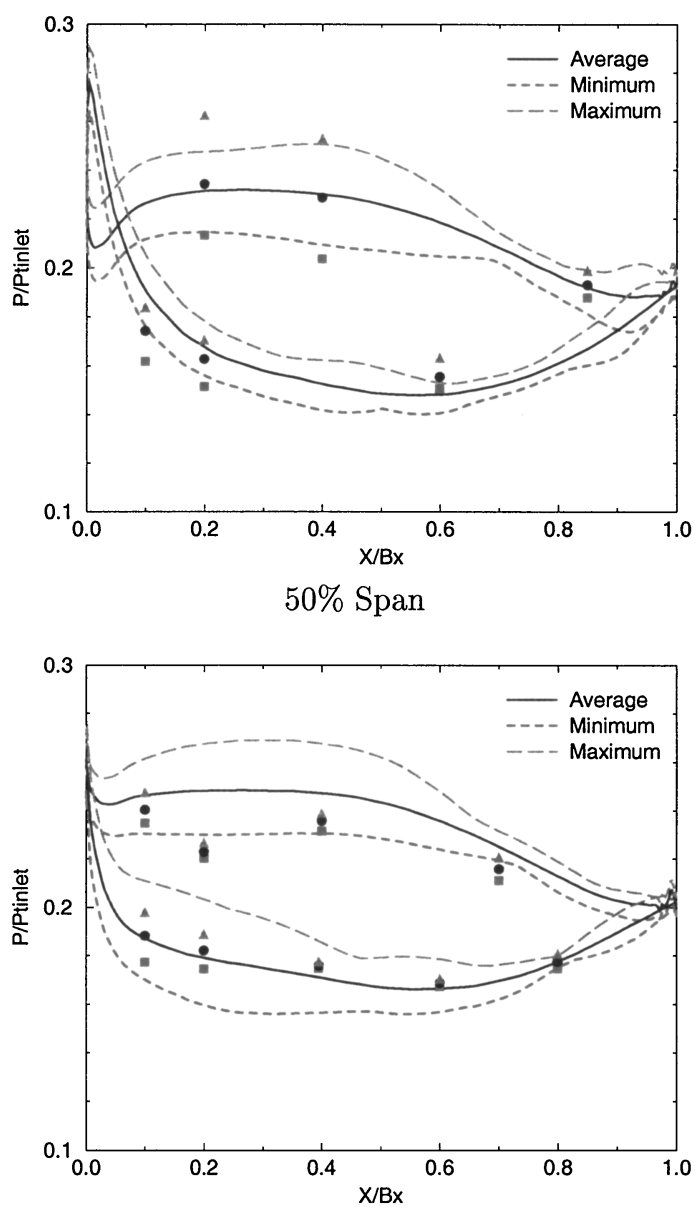

$90 \%$ Span

FIGURE 3

Low-pressure turbine vane pressure distributions. (Note different pressure scale compared to blade.)

agreement with the data but the computed pressure envelope is again too large compared with the data for most of the surface. The unsteady pressure envelope at this spanwise position is significantly influenced by the blade tip-clearance flow. The 
over-prediction in the unsteady pressure levels at the $90 \%$ span location indicates that the computed blade tip-vortex flow does not mix enough as it convects along the endwall through the transition duct.

Comparison of Figures 2 and 3 shows that the largest unsteady pressure envelopes are on the blade suction surface near the leading edge due to the interaction with the first vane. The magnitude of the unsteady pressure in this region of the blade is approximately twice as large as that anywhere else on the blade or on the downstream low-pressure turbine vane. The interaction between the blade and the downstream vane is weaker than that between the first vane and the blade primarily due to the much larger axial spacing between the blade and downstream vane as well as the radius change between the high- and low-pressure turbine.

\section{Unsteady Flow Characteristics}

The unsteady pressure signals on the blade surfaces are postprocessed using Fast Fourier Transforms (FFT) to obtain information in the frequency domain. Clark et al. (2000) showed that the fundamental and first harmonic of the pressure make up over $90 \%$ of the pressure envelope over most of the surface. Figure 4 shows contour plots of the normalized amplitude of the unsteady pressure at the fundamental (vane-pass) frequency (36E) and the first harmonic (72E). The pressure amplitudes are plotted as a percent of the upstream total pressure. Each blade is unwrapped along the trailing edge line for plotting purposes. The locations of the Kulites used to measure the unsteady pressure are also shown in these figures.

Figure 4 shows that the peak pressure amplitude of the fundamental (36E) and first harmonic (72E) frequencies are located on the pressure side of the blade near $10 \%$ span and on the suction side just downstream of the leading edge. These high unsteady pressures are due to the interaction between the blade and upstream vane. Moderately high pressure amplitudes also exist at both frequencies on the suction side of the blade between $80 \%$ axial chord and the trailing edge and between the hub and $70 \%$ span. This region of high unsteady pressure results from the interaction between the blade and the downstream transition duct and vane. This interaction will be discussed further below.

A comparison between the computed and experimental unsteady surface spectrum at points on the blade surface where the amplitude of the unsteady pressure is greatest is shown in Figure 5. The two points selected on the pressure side are at $10 \%$ span around mid-axial chord where the unsteady pressure is greatest due to the interaction with the upstream vane. The computed pressure spectrum are in good agreement with the experimental data. On the pressure side of the blade, the maximum differences between the computed and experimental amplitudes of the fundamental and first harmonic are around $0.1 \%$ of the inlet total pressure. This represents a 10-15\% difference between the computed and the experimental amplitudes. The amplitude of the fundamental mode is slightly under-predicted whereas the amplitude of the first harmonic is slightly over-predicted.
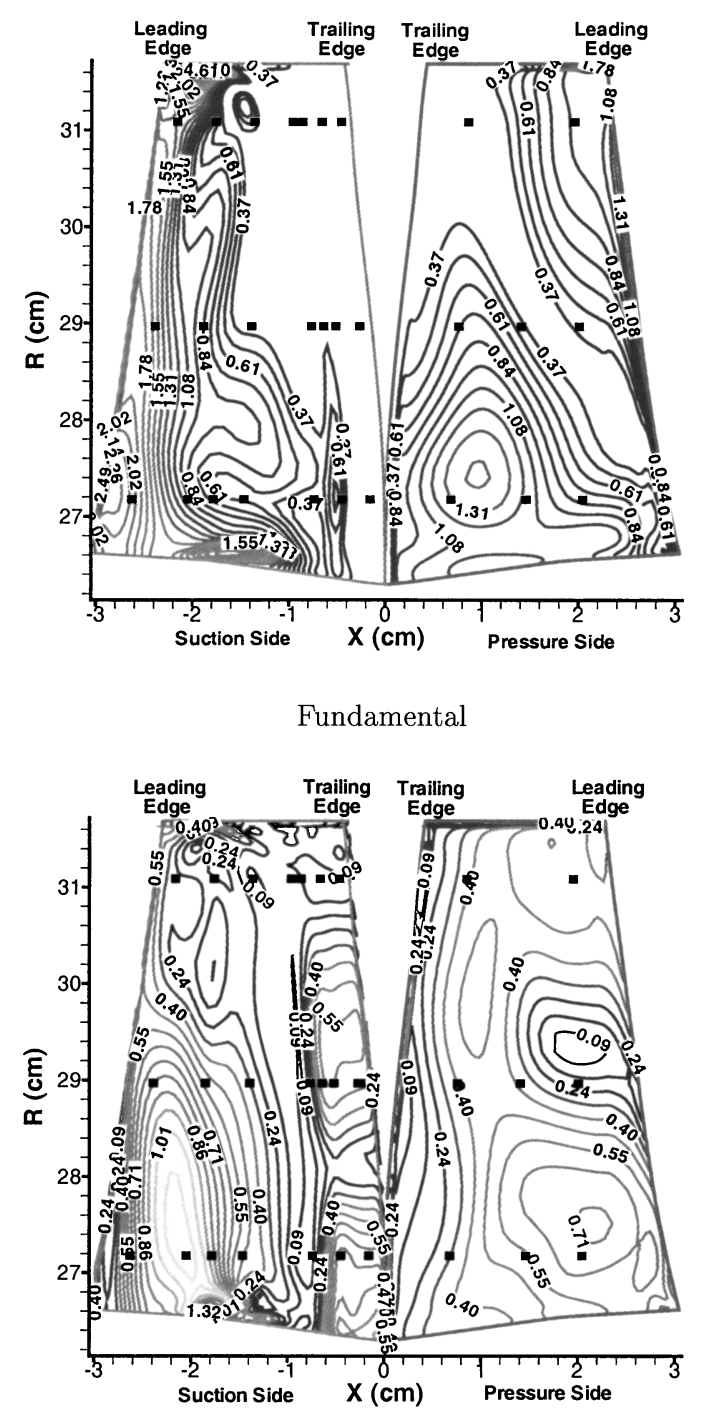

1st Harmonic

FIGURE 4

High-pressure turbine blade computed fundamental and 1st harmonics of pressure (experimental data acquisition locations shown as black squares).

The two points selected on the suction surface of the blade for comparison are again located at $10 \%$ span and at 30 and $75 \%$ axial chord. The point at $30 \%$ axial chord has high unsteady pressure due to the interaction with the upstream vane. The point at $75 \%$ axial chord is located downstream of the blade throat in the region of interaction with the adjacent blade's pressure-side trailing edge shock and the reflected waves from the downstream vane. Figure 5 shows that the agreement between the computed and experimental pressure spectrum at the $30 \%$ axial chord position is fairly good with the maximum difference being around $0.25 \%$ of the inlet total pressure. This represents a difference of $20 \%$ between the computed and experimental amplitude. Once again, the amplitude of the fundamental mode is under-predicted 


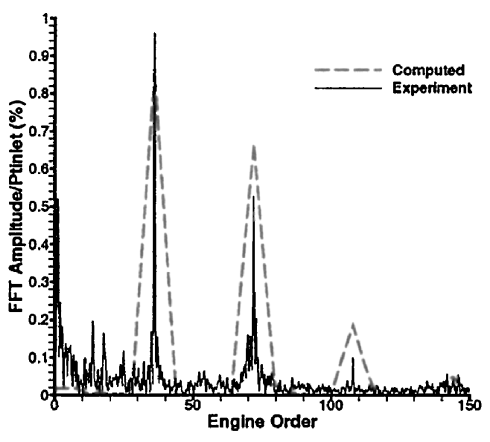

10\% Span, 30\% Axial Chord, Pressure Side (1st Kulite from Leading Edge)

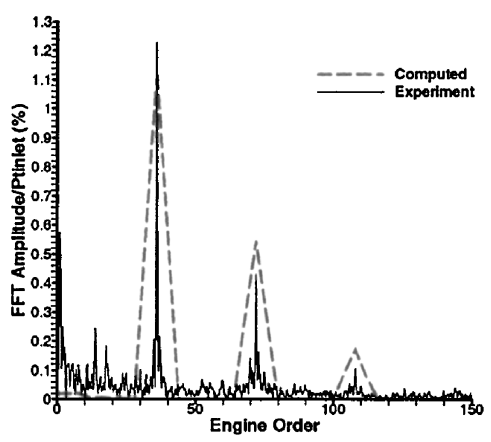

$10 \%$ Span, $50 \%$ Axial Chord, Pressure Side (2nd Kulite from Leading Edge)

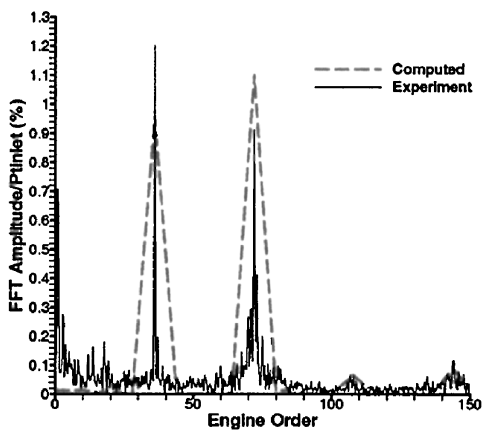

10\% Span, 30\% Axial Chord, Suction Side (2nd Kulite from Leading Edge)

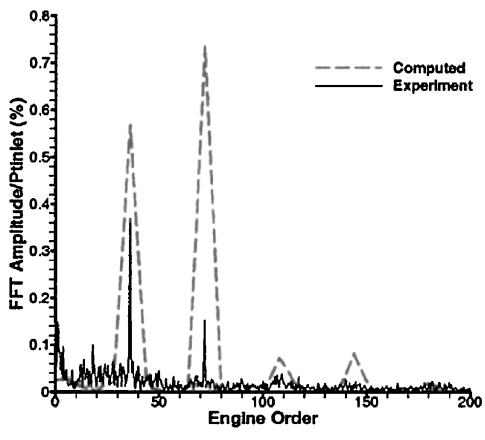

$10 \%$ Span, $75 \%$ Axial Chord, Suction Side) (6th Kulite from Leading Edge)

FIGURE 5

Computed and experimental spectrum on blade surface. whereas the amplitude of the first harmonic is over-predicted. Larger differences exist between the computed and experimental amplitudes at the $75 \%$ axial chord position, however. Here, the simulation over-predicts the fundamental mode amplitude by $0.2 \%$ of the inlet total pressure which corresponds to a $50 \%$ greater amplitude. The difference in the amplitude of the first harmonic is much larger ( $0.6 \%$ of the inlet total pressure) with the simulation significantly over-predicting the amplitude.

Comparison between the current predicted blade unsteady surface pressure spectrums and those of Clark et al. (2000) at the experimental data locations show that the two independent predictions are in good agreement. As pointed out above in the discussion of the time-averaged pressure and pressure envelopes for the blade, the mismatches between both predictions and the experimental unsteady blade surface data at some locations on the blade are likely due to different blade shapes and/or the inlet profiles than the experiment. However, as pointed out by Clark et al. (2000) both predictions would generally lead to conservative blade designs as a result of their over-prediction of the pressure amplitudes.

The pressure amplitude at the fundamental (56E) and first harmonic (112E) frequencies on the surface of the downstream low-pressure turbine vane is shown in Figure 6. Again, the surface of the airfoil is unwrapped around the trailing edge and the locations of the Kulites used to measure the unsteady surface pressure are shown similar to the blade. Figure 6 shows the peak unsteady pressure amplitude to be located on the pressure side between the leading edge and mid-axial chord between the hub and mid-span. This region of high unsteady pressure arises from the interaction of the blade trailing edge shock system with the low-pressure turbine vane. Another region of high unsteady pressure is located near the tip leading edge on the suction side of the airfoil. This high unsteady pressure is due to the interaction of the vane with the blade wake/tip-vortex.

A comparison between the computed and experimental pressure spectrum at points on the vane surface are shown for the first time in Figure 7. The points chosen for comparison were located in the regions of peak unsteady pressure located on the pressure surface at $10 \%$ span between 20 and $40 \%$ axial chord and on the suction surface at $90 \%$ span near the leading edge. The agreement between the computed and experimental amplitude of the fundamental mode of unsteady pressure is fair with a difference varying between 0.25 and $0.8 \%$ of the upstream total pressure. This corresponds to an over-prediction in the amplitude of the fundamental mode ranging from 45 to $160 \%$, however, with the largest difference at the $90 \%$ span, $20 \%$ axial chord position. At this position, the computation predicts a stronger interaction with the blade wake/tip-vortex. The computed amplitude of the first harmonic is in good agreement with the experiment, however. On the pressure surface of the low-pressure turbine vane at the $10 \%$ span position, the simulation consistently over-predicts the amplitudes and interaction due to the blade trailing edge shock system. This over-prediction is consistent with the overpredicted amplitudes on the blade suction surface downstream 


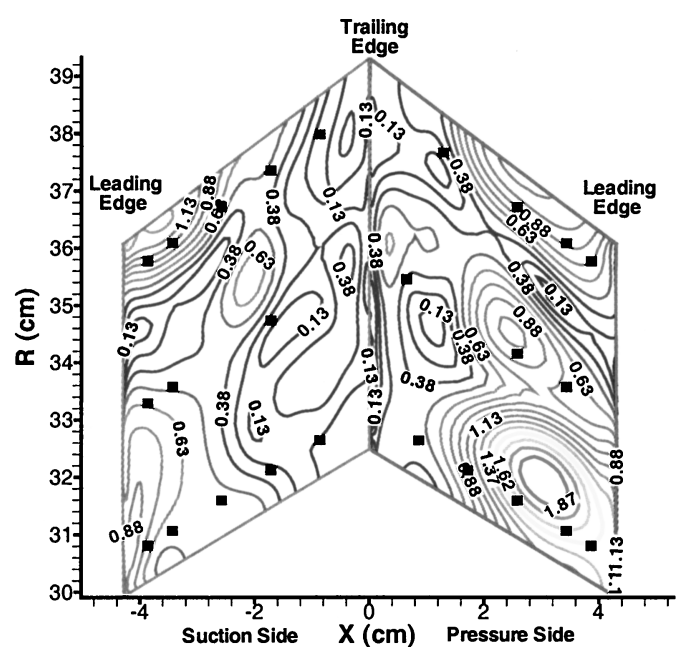

Fundamental

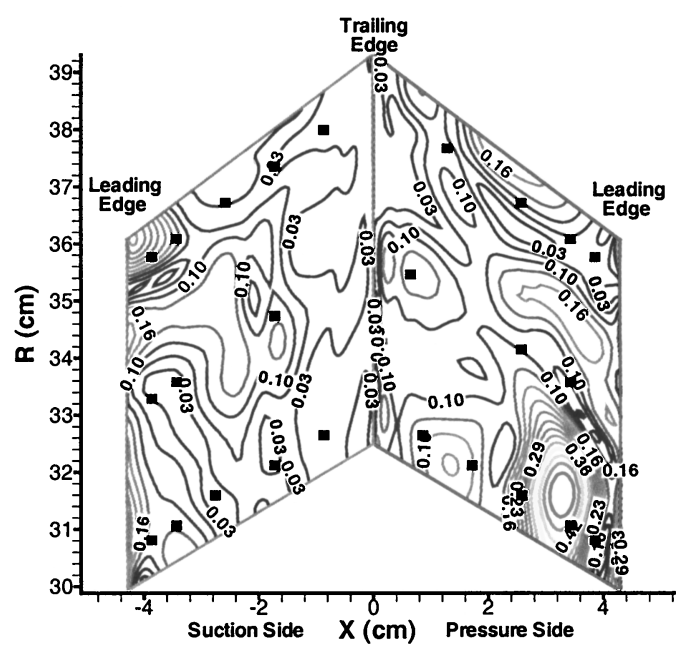

1st Harmonic

FIGURE 6

Low-pressure turbine vane computed fundamental and 1st harmonics of pressure (experimental data acquisition locations shown as black squares).

of the throat at $10 \%$ span which are affected by the reflection of the blade trailing edge shocks from the low-pressure turbine vane. Although the amplitude of the unsteady pressure is overpredicted in the regions of high interaction at the 10 and $90 \%$ span positions (near the endwalls) on the second vane, Figure 3 shows that the unsteady pressure amplitude at mid-span agrees very well with the experiment.

Comparison between the current low-pressure turbine vane unsteady surface pressure spectrums and those of Clark et al. (2001) at the experimental data locations show that agreement with the unsteady experimental data has generally improved in the current investigation as a result of the increased computational grid density and use of Wilcox's (1998) turbulence

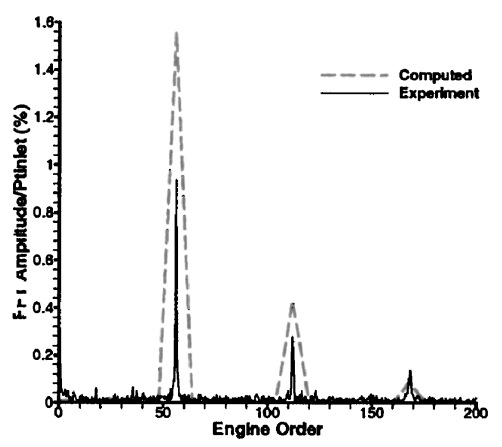

$10 \%$ Span, $20 \%$ Axial Chord, Pressure Surface (2nd Kulite from Leading Edge)

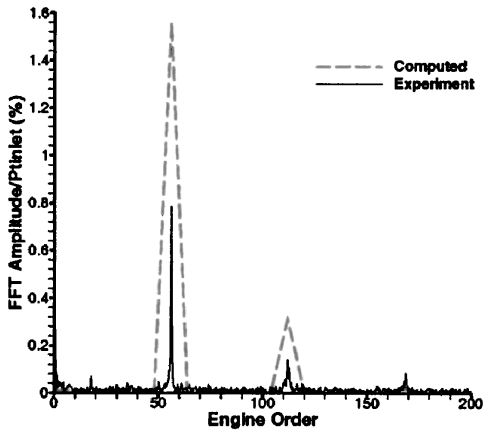

10\% Span, 40\% Axial Chord, Pressure Surface (3rd Kulite from Leading Edge)

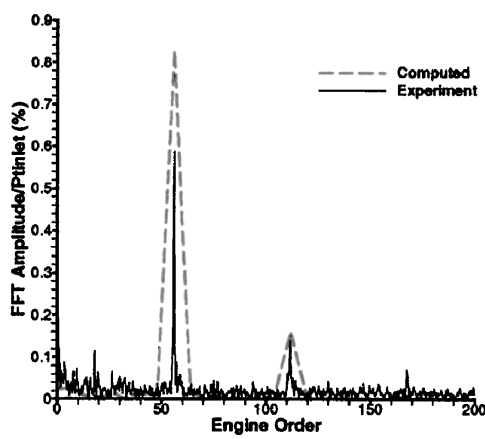

90\% Span, $10 \%$ Axial Chord, Suction Surface (1st Kulite from Leading Edge)

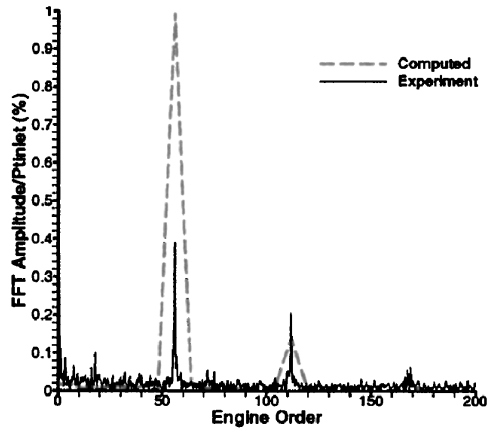

90\% Span, $20 \%$ Axial Chord, Suction Surface (2nd Kulite from Leading Edge)

FIGURE 7

Computed and experimental spectrum on second vane surface. 

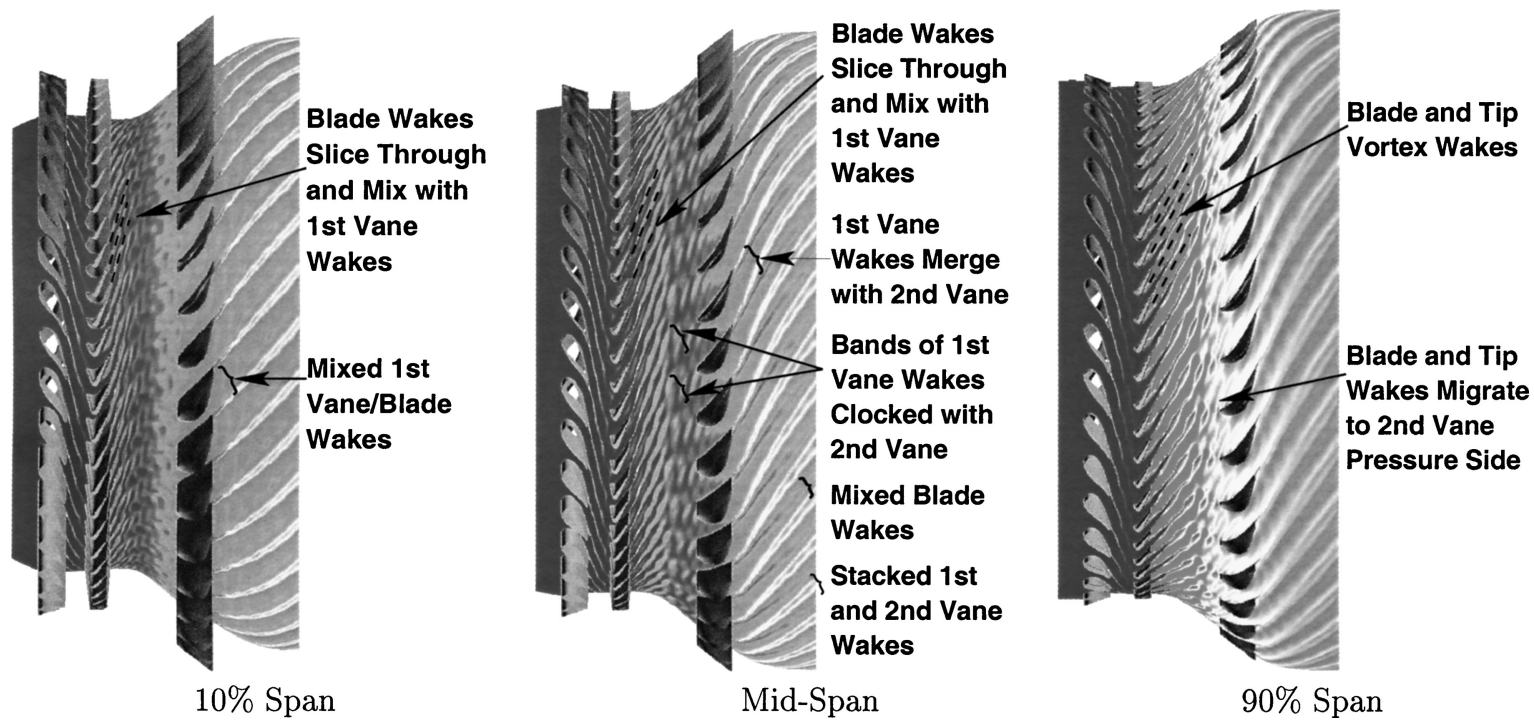

FIGURE 8

Computed instantaneous entropy contours at 10,50, and 90\% span.

model. However, as pointed out above in the discussion of the time-averaged pressure and pressure envelopes, further improvement in the agreement may be possible through the inclusion of blade hub leakages. Also, recent investigations (Knight et al., 2002; Liou et al., 2000; Dolling, 2001; Sinha et al., 2004) have shown that two-equation turbulence models, like that used in the current investigation, often fail to accurately predict the pressure field and boundary-layer characteristics for shock/boundary-layer interactions, especially when the flow is unsteady. So further improvement in the predicted unsteady surface pressure spectrum for this transonic turbine may also be possible with appropriate modifications to the turbulence model for the unsteady, shock/boundary-layer flow. Further research and numerical simulations are necessary to resolve these issues.

Figures 8 and 9 illustrate the various phenomena that lead to the unsteady interactions between the three components, especially those between the blade, transition duct, and low-pressure turbine vane. These figures show the instantaneous entropy and pressure contours at the $10 \%$ span, mid-span, and $90 \%$ span planes. Figure 9 shows the pressure jump associated with the blade trailing edge shock system. As the blade rotates, the blade trailing edge shock extends to the downstream vane where it strikes and sweeps forward along the vane pressure side. As

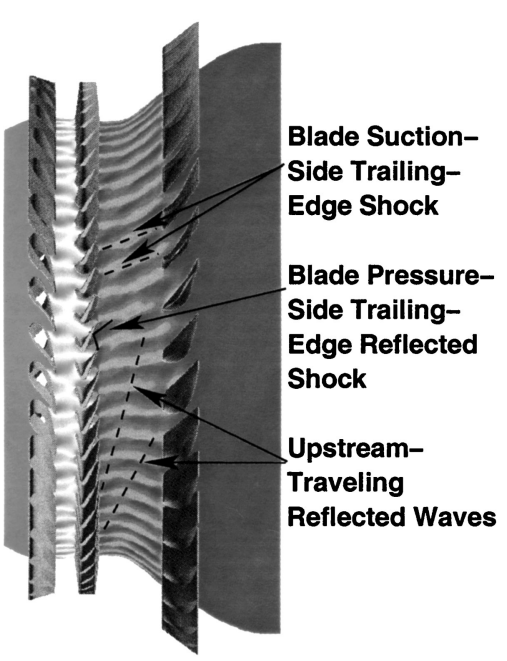

$10 \%$ Span
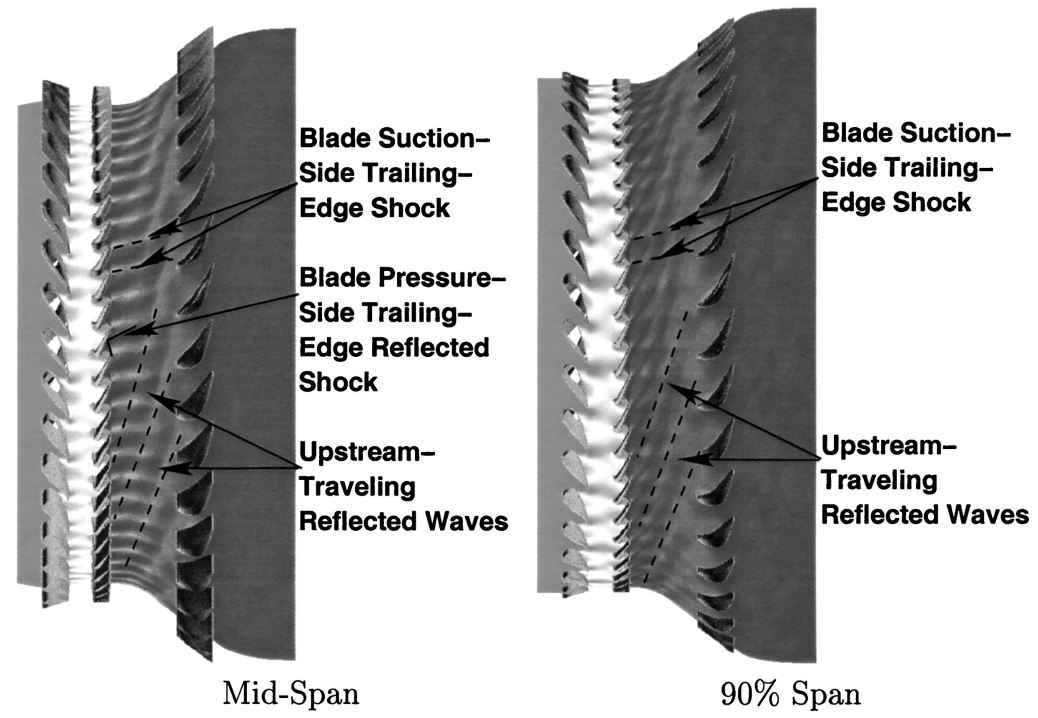

FIGURE 9

Computed instantaneous pressure contours at 10, 50, and 90\% span. 
shown in Figure 4, the blade tip radius is approximately $31.6 \mathrm{~cm}$. The shock sheet generated from the blade trailing edge shock system propagates downstream at a constant radius so that only radial positions below $31.6 \mathrm{~cm}$ are directly struck by this shock. The peak unsteady pressure on the vane pressure side is located near the leading edge and around $31.6 \mathrm{~cm}$ for this reason. Some moderate levels of unsteady pressure are also located on the vane near and above mid-span. The source of this unsteady pressure is the blade trailing edge shock reflections off of the transition duct into the vane. Another area of high unsteady pressure is at the vane tip leading edge near the $90 \%$ span location. The unsteady pressures at this location are attributed to the blade tip vortex that migrates inboard somewhat as it convects through the transition duct as previously described.

As shown in Figure 8, the wakes at mid-span from the upstream first vane mix with the blade wakes into bands of high entropy that are clocked to the leading edge of the downstream low-pressure turbine vane. Several investigations (Dorney et al., 2001; Cizmas and Dorney, 1998; Dorney and Sondak, 2000) into blade wake clocking in multi-stage turbomachinery suggest that the current vane wake clocking could provide optimal performance. The blade wakes strike the downstream vane at the leading edge and, along with the first vane wakes, tend to migrate to the suction side of the low-pressure turbine vane. At the 10 and $90 \%$ span locations, the secondary and tip vortex flows mix with the first vane wakes more thoroughly such that the entropy going into the second vane is more uniform. The blade tip vortex strikes the low-pressure turbine vane leading edge and migrates to the pressure side of the vane. There are typically two blade/tip vortex wakes in the low-pressure turbine vane passage at any instant in time.

Inspection of Figure 9 reveals that the shock wave emanating from the trailing edge of the blade impacts the pressure side of the low-pressure turbine vane and sweeps forward on that surface as the rotor revolves around the turbine axis. This physical process is evident in the experimental data and is captured very well by the simulation, even though there are some misses in the predicted magnitudes of the unsteady shock interaction on the downstream vane pressure and suction sides near the endwalls. Figure 10 is a plot of results from 2-point spacetime correlations (Ifeachor and Jervis, 1996) of the time-resolved pressure field evaluated on the surface of the low-pressure turbine vane pressure side at $10 \%$ span. The figure is presented as a set of shock trajectories along the second vane pressure side with the sensor at $40 \%$ axial chord acting as the reference, and both experimental and predicted shock trajectories are shown. To obtain a single point on each of the trajectories, the crosscovariance coefficient between the signal of the reference sensor and another sensor (e.g., that at $20 \%$ axial chord) is calculated for both positive and negative time lags on a range from zero to an absolute value greater than the period of blade passing. The time-lag at which the maximum cross-covariance coefficient occurs is taken to be the propagation time of the shock as it travels

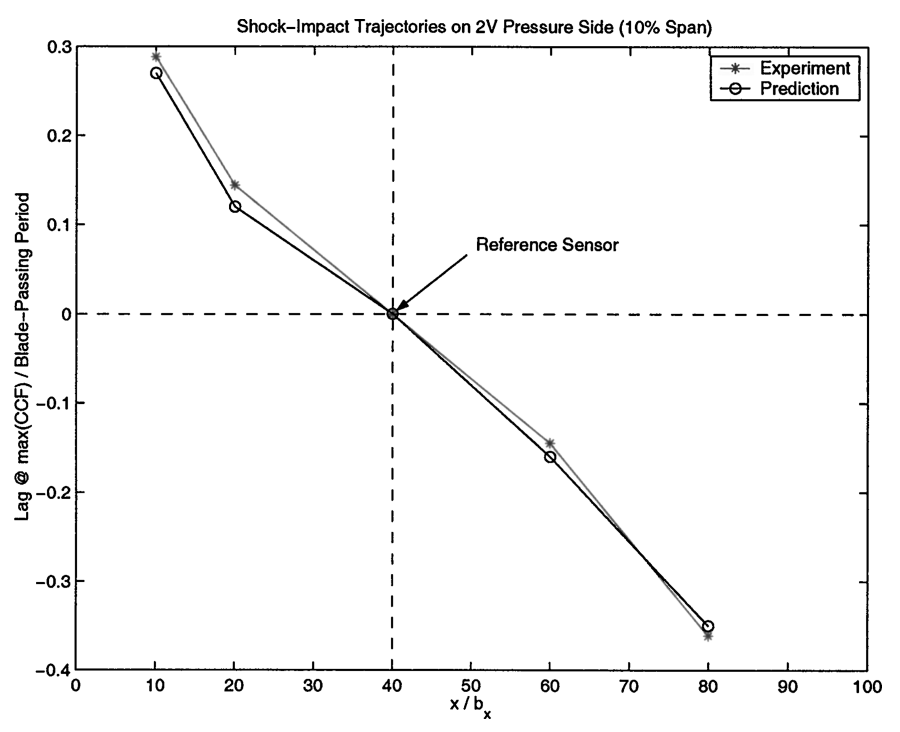

FIGURE 10

Computed and experimental shock trajectory on second vane pressure surface.

between the reference sensor and the local sensor. That time is then normalized by the period of blade passing and plotted on the ordinate of Figure 10, while the percent axial chord is plotted on the abscissa.

The trajectory of the blade trailing-edge shock along the second vane pressure side is very well predicted, and this gives credence to the physical description of the flow-field embodied in Figure 9. This level of agreement also implies that the blade shock angle is well captured by the simulation. Also, the measured time-mean loading on the blade suction side near the trailing edge, as shown in Figure 2, is well predicted by the simulation, and that is indicative of the trailing edge free-stream Mach number and blade shock strength. Again, Figure 3 shows that the over-prediction of the pressure envelopes/amplitudes occurs primarily at the 10 and $90 \%$ span locations near the endwalls whereas the mid-span unsteadiness is predicted quite well. Therefore, the blade shock angle and strength are well predicted and the physics of the blade/second vane shock interaction is well captured by the simulation, but the magnitude of that interaction near the endwalls tends to be over-predicted. A similar over-prediction in the second vane unsteady pressure amplitudes was also observed in the simulations of Clark et al. (2000). A likely reason for the discrepancy in both simulations is the inability of the turbulence models to "react" properly to the inclined shock (i.e., unsteady shock/boundary layer interaction) as it penetrates the low-pressure turbine vane pressure-side boundary layer/secondary flow and the clocked first vane/blade wakes, and/or in the mixing of the endwall flows. Another point to note is that the gradients in the unsteady pressure are also quite large around the islands of peak unsteady pressure amplitude. As a result, a small error in the predicted size and location 
TABLE 1

Comparison of Computed Relative Total Pressure Loss Coefficients

\begin{tabular}{lcc}
\hline Blade-row & $\begin{array}{c}\text { Predicted } \\
\text { time-averaged } \\
\text { relative loss }\end{array}$ & $\begin{array}{c}\text { Predicted } \\
\text { steady } \\
\text { relative loss }\end{array}$ \\
\hline HPT vane & 0.043 & 0.029 \\
HPT blade & 0.104 & 0.094 \\
LPT vane & 0.040 & 0.038 \\
\hline
\end{tabular}

of the peak regions can translate into a large difference between the computed and experimental unsteady pressure amplitude.

\section{AERODYNAMIC LOSSES}

A comparison between the time-averaged, relative total pressure loss and the relative total pressure loss computed from the steady-flow analysis using the TFLO procedure with interblade mixing planes is given in Table 1 . The differences between the two computed loss levels are an indication of the effect of the unsteady flow on the aerodynamic losses for each bladerow. The relative total pressure loss coefficient in Table 1 is given as the change in relative total pressure across a given blade-row non dimensionalized by the inlet total pressure to that blade-row, $\Delta\left(\mathrm{P}_{\mathrm{T}}\right) / P_{\text {Tin }}$. The total pressure was not measured in the experiment except at the inlet to the high-pressure turbine vane and at the exit of the low-pressure turbine vane due to space and flow constraints in the rig. Therefore, the relative total pressure loss for each blade-row could not be obtained experimentally.

Table 1 shows that the predicted losses from the time-average of the unsteady simulation are all larger than those from the steady, multi-stage simulation. The time-averaged loss for the first vane is significantly larger due to the interaction with the blade and the reflected shocks/waves described above. The timeaveraged loss for the blade as well as the downstream lowpressure turbine vane are only slightly greater than the steady, multi-stage values, however. This implies that although the interaction between the blade and the low-pressure turbine vane increases the unsteady pressure levels in both blade-rows, it does not seem to result in significant aerodynamic loss penalties. This is probably due to the large axial spacing between these two blade-rows.

\section{CONCLUSIONS}

The use of large-scale, massively parallel computer systems is making it possible to simulate true blade counts over increasing numbers of blade-rows in a turbomachine. New ReynoldsAveraged Navier-Stokes solution procedures, such as TFLO, are continuing to be developed to take advantage of these computer systems, and to be validated and demonstrated for large-scale turbomachinery applications. Further research and development of these massively parallel computer systems and simulation

tools should be performed to make computations, such as the current one, routine and affordable.

In the current investigation, interrogation of the computation along with the experiment has uncovered new multi-component interaction flow mechanisms that are unique to transonic, highpressure turbine stages. These interactions between the highand low-pressure turbine and transition duct affect the unsteady pressure field as well as the aerodynamic performance. These interactions include shock and pressure waves that propagate from the blade to the downstream components and reflect back upstream to the blade itself.

The current investigation has also shown that the computed time-averaged pressure distributions and unsteady pressure envelopes generally agree well with experimental data. In addition, the predicted interaction between the high-pressure vane and blade agrees well with the data. Similar agreement exists for the blade/low-pressure turbine vane interaction around midspan. However, the unsteady pressure envelopes and unsteady pressure amplitudes on the low-pressure turbine vane are generally over-predicted near the endwalls suggesting room for further improvement in endwall turbulence and leakage modeling. Finally, comparison between the losses computed from the timeaveraged, unsteady and steady simulations show that the largest loss in relative total pressure resulting from the unsteady interaction occurs in the high-pressure turbine vane due to the close axial spacing from the blade.

\section{NOMENCLATURE}

$\mathrm{Bx}$

Corrected Flow Parameter

Corrected Speed Parameter

$P$

$\mathrm{Pt}$

$T$

$\mathrm{Tt}$

$\mathrm{R}$

RPM

X

\section{Subscripts}

inlet axial chord of airfoil section $(\mathrm{cm})$

$\dot{m} \sqrt{T t_{\text {in }}} / P t_{\text {in }}$

$R P M / \sqrt{T t_{\text {in }}}$

pressure $(\mathrm{Pa})$

stagnation pressure $(\mathrm{Pa})$

temperature $(\mathrm{K})$

stagnation temperature $(\mathrm{K})$

radius $(\mathrm{cm})$

blade rotational velocity (rotations

per minute)

axial distance $(\mathrm{cm})$

\section{REFERENCES}

Arnone, A., Marconcini, M., Pacciani, R., Schipani, C., and Spano, E. 2001. Numerical investigation of airfoil clocking in a three-stage low pressure turbine. ASME Paper 2001-GT-0303.

Baldwin, B., and Lomax, H. 1978. Thin-layer approximation and algebraic model for separated turbulent flows. AIAA Paper 1978-0257.

Busby, J. A., Davis, R. L., Dorney, D. J., Dunn, M. G., Haldeman, C. W., Abhari, R. S., Venable, B. L., and Delaney, R. A. 1998. Influence of vane-blade spacing on transonic turbine stage aerodynamics, Part II: Time-resolved data and analysis. ASME 98-GT-482. 
Chen, J. P., Celestina, M. L., and Adamczyk, J. J. 1994. A new procedure for simulating unsteady flows through turbomachinery blade rows., ASME Paper 94-GT-151.

Cizmas, P., and Dorney, D. J. 1998. Parallel computation of turbine blade clocking. AIAA Paper 98-3598.

Clark, J. P. 2001. Private communication.

Clark, J. P., Stetson, G. M., Magge, S. S., Ni, R. H., Haldeman, C. W., and Dunn, M. G. 2000. The effect of airfoil scaling on the predicted unsteady loading on the blade of a 1 and 1/2 stage transonic turbine and a comparison with experimental results. ASME Paper 2000-GT0446.

Dolling, D. S. 2001. Unsteadiness of shock-induced turbulent separated flows-Some key questions. AIAA Paper 2001-2708.

Dorney, D. J., Croft, R. R., Sondak, D. L., Stang, U. E., and Twardochleb, C. Z. 2001. Computational study of clocking an embedded stage in a 4-stage industrial turbine. ASME Paper 2001-GT0509.

Dorney, D. J., and Sondak, D. L. 2000. Three-dimensional simulations of airfoil clocking in a 1-1/2 stage turbine. AIAA Paper 2000 3359.

Dring, R. P., Joslyn, H. D., Hardin, L. W., and Wagner, J. H. 1982. Turbine rotor-stator interaction. ASME Journal of Engineering for Power 104:729-742.

Dunn, M. G., Moller, J. C., and Steele, R. C. 1989. Operating point verification for a large shock tunnel test facility. WRDC-TR2027.

Giles, M. B. 1990. Stator/rotor interaction in a transonic turbine. AIAA Journal of Propulsion and Power 6:621-627.

Gombert, R., and Hohn, W. 2001. Unsteady aerodynamical blade row interaction in a new multistage research turbine-Part 1: Experimental investigation. ASME Paper 2001-GT-0306.

Hohn, W., Gombert, R., and Kraus, A. 2001. Unsteady aerodynamical blade row interaction in a new multistage research turbine-Part 2: Numerical investigation. ASME Paper 2001-GT-0307.

Ifeachor, E. C., and Jervis, B. W. 1996. Digital Signal Processing, New York: Addison-Wesley.

Jameson, A. 1991. Time dependent calculations using multigrid, with applications to unsteady flows past airfoils and wings. AIAA Paper
91-1596, AIAA 10th Computational Fluid Dynamics Conference, Honolulu, HI.

Jorgenson, P. C. E., and Chima, R. 1990. An Explicit Runge-Kutta method for unsteady rotor/stator interaction. AIAA Paper 90-2408.

Knight, D., Yan, H., Panaras, A., and Zheltovodov, A. 2002. RTO WG 10: CFD validation for shock wave turbulent boundary layer interactions. AIAA Paper 2002-0437.

Liou, W. W., Huang, G., and Shih, T-H. 2000. Turbulence mocel assessment for shock wave/turbulent boundary layer interaction in transonic and supersonic flows. Computers and Fluids 29:275-299.

Rai, M. M. 1987. Navier-Stokes simulations of rotor-stator interaction using patched and overlaid grids. AIAA Journal of Propulsion and Power 3:387-396.

Rao, K. V., Delaney, R. A., and Dunn, M. G. 1994. Vane-blade interaction in a transonic turbine, Part 1: Aerodynamics. ASME Journal of Propulsion and Power 10(3):305-311.

Reinmoller, U., Stephan, B., Schmidt, S., and Niehuis, R. 2001. Clocking effects in a 1.5 stage axial turbine — steady and unsteady experimental investigations supported by numerical simulations. ASME Paper 2001-GT-0304.

Reynolds, W. C., Fatica, M., and Alonso, J. J., Eds. 2001. 2001 annual technical report-Department of energy accelerated strategic computing initiative, Stanford University Report.

Sharma, O. P., Pickett, G. F., and Ni, R. H. 1992. Assessment of unsteady flows in turbines. ASME Journal of Turbomachinery 114:79-90.

Sinha, K., Mahesh, K., and Candler, G. V. 2004. Modeling the effect of shock unsteadiness in shock-wave/turbulent boundary layer interactions. AIAA Paper 2004-1129.

Takahashi, R., and Ni, R. H. 1991. Unsteady hot streak simulation through 1-1/2 stage turbine. AIAA Paper 91-3382.

Wilcox, D. C. 1998. Turbulence Modeling for CFD, La Cañada, CA: DCW Industries, Inc.

Yao, J., Davis, R. L., Alonso, J. J., and Jameson, A. 2002. Massively parallel simulation of the unsteady flow in an axial turbine stage. AIAA Journal of Propulsion and Power 18(2):465-471.

Yao, J., Jameson, A., Alonso, J. J., and Liu, F. 2001. Development and validation of a massively parallel flow solver for turbomachinery flows. AIAA Journal of Propulsion and Power 17(3):659-668. 

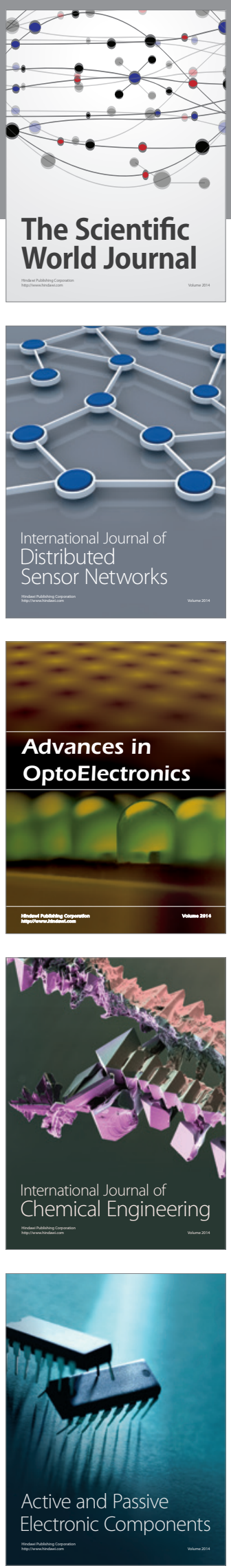
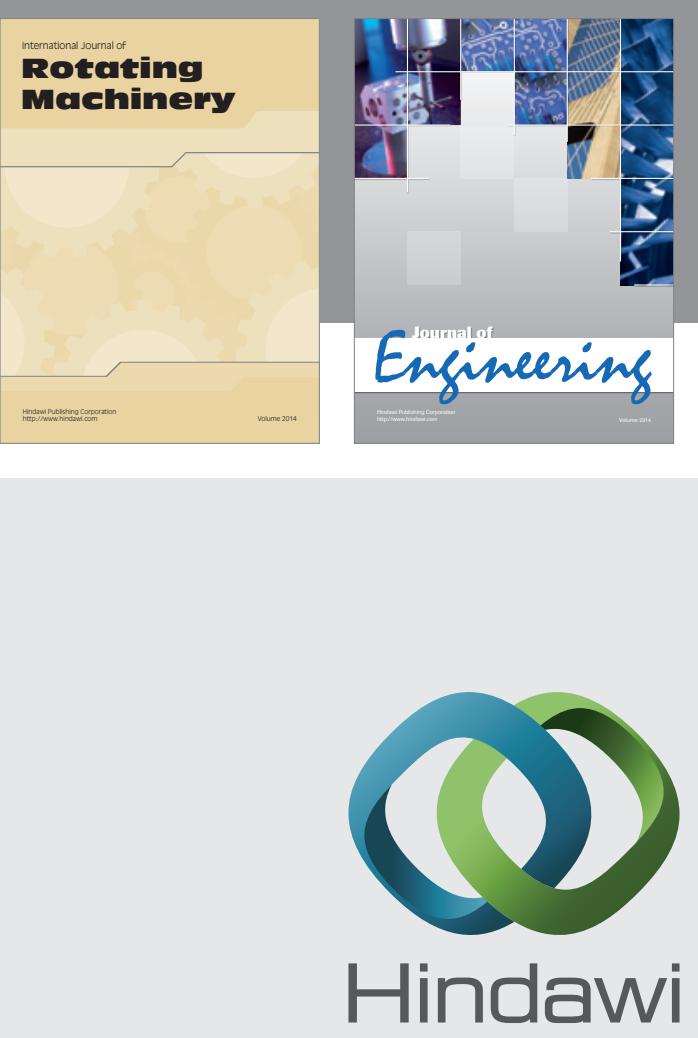

Submit your manuscripts at

http://www.hindawi.com
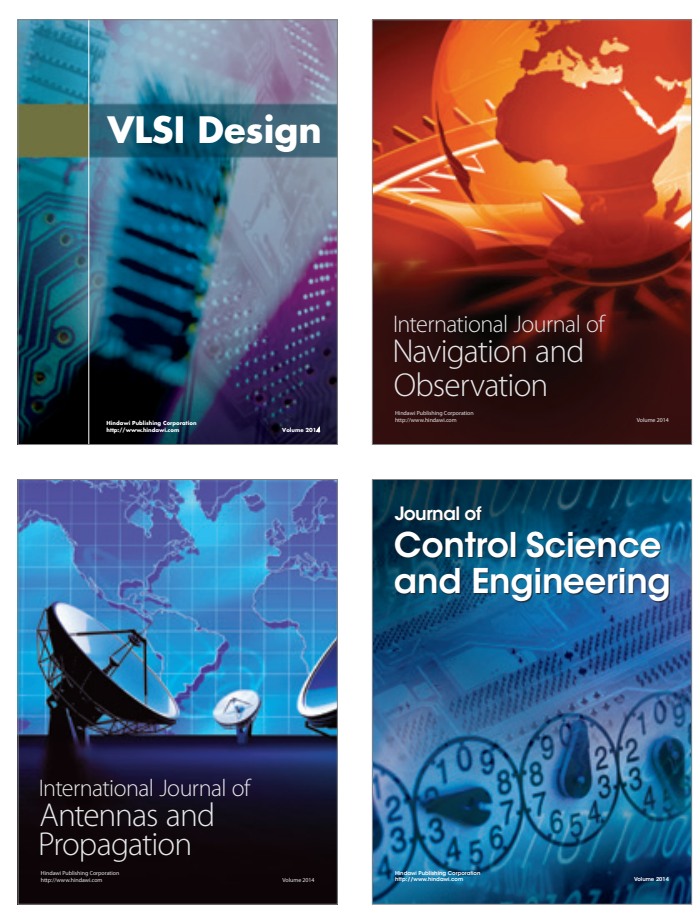
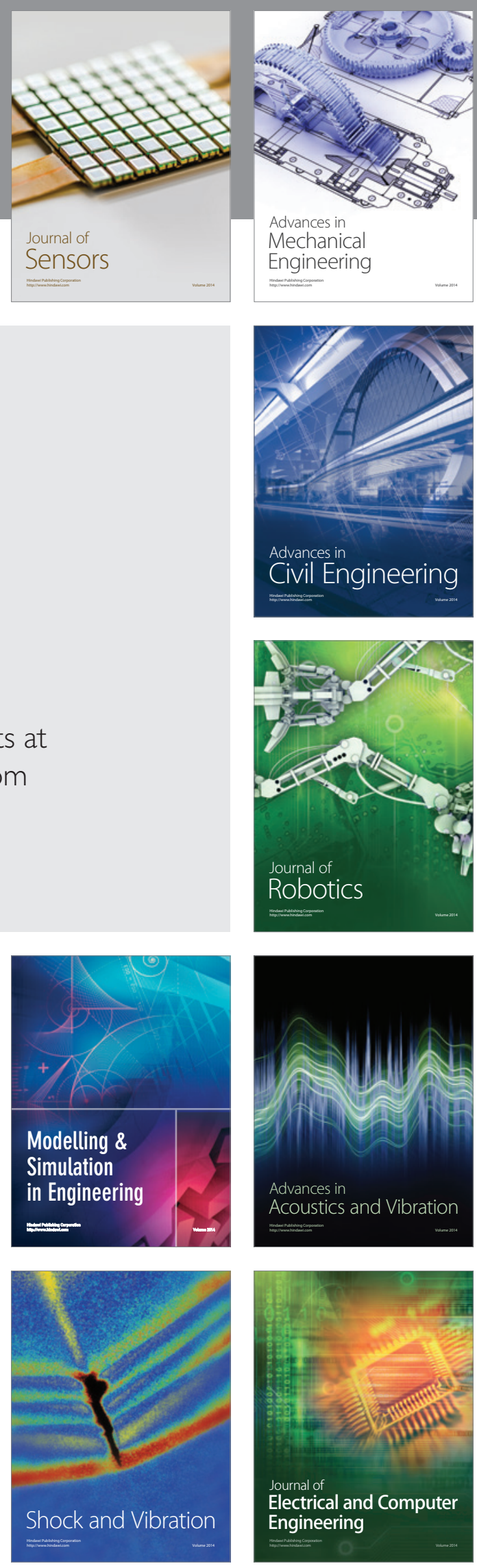